\title{
Cloud Based Health Monitoring and Abnormality Detection using Smart Mirror
}

\author{
D. Prabha' ${ }^{1}$ M.S. Karthika' and P. Manivanan ${ }^{2}$ \\ 'Department of Computer Science and Engineering, Sri Krishna College of Engineering and Technology, \\ Kuniamuthur-641008, Coimbatore, Tamil Nadu, India; prabha@skcet.ac.in, 17epcs008@skcet.ac.in \\ ²Department of Computer Science and Engineering, Park College of Technology, Kaniyur -641659, \\ Coimbatore, Tamil Nadu, India; pctmani@gmail.com
}

\begin{abstract}
Background/Objective: To provide an efficient predictive technique to foresee future workload as well as to handle the resources efficiently by performing hybrid auto scaling for Cloud applications. Cloud applications might expertise completely different workload at different times, automatic provisioning has to work with efficiency at any point of time. Auto scaling is a feature of cloud computing that potentially scale the resources in line on demand. Considering this expectation, they are generally categorized into Reactive scaling which adds or reduces resources based on a fixed threshold value. The predictive scaling is used provide necessary scaling actions beforehand. Methods/Statistical Analysis: To perform the hybrid auto scaling (reactive plus predictive auto scaling), a time series technique should be used. Auto-regressive Moving Average (ARMA) model, the Exponential Smoothing (ES) model, the Autoregressive model (AR), the Moving Average model (MA) and the Trend- Adjusted Exponential Smoothing (TAES), Auto Regressive Integrated Moving Average (ARIMA) Time-series model, Naïve bayes algorithm, Recurrent Neural Network- Long Short Term Memory (RNN-LSTM), Independent Recurrent Neural Network (IndRNN) are time series techniques used to foresee the future workload. To find the effectiveness of predictive techniques, Mean Absolute Error (MAE), Mean Squared Error (MSE) and Mean Absolute Percentage Error (MAPE) performance metrics are evaluated. Findings: Based on the evaluation, IndRNN gives the minimum error rate. IndRNN is used to predict the future resource requisites in order to ascertain adequate resource are available ahead of time. Application: The predicted result from IndRNN method is integrated on private cloud to autoscale the resources for cloud applications.
\end{abstract}

Keywords: Abnormality, Cloud, Health Monitoring, Raspberry Pi, Sensor, Smart Mirror, Two Way Mirror

\section{Introduction}

There are different techniques for health monitoring. Different types of sensors are used for such purposes. Early health monitoring techniques are clinical, where in with the help of trained therapist; an individual could learn to control specific physiological functions by changing the thoughts and perceptions that produce them. But it needs to travel to the corresponding place for the advices. Today, the technology changed to which the individuals can become aware of their physiological functions using biomedical sensors with the help of computer systems. Now the sensors are added to the devices like mobile phones and watches. It is very useful for the persons who are able to use such devises.

But the old aged people who are unaware of using such devices need more such health monitoring systems. So this paper introduces an effective health monitoring system using mirrors which is a device used by all kind of people irrespective of their ages.

The biomedical sensors prxesent within the mirror will collect the physiological features and the collected data will be sent to the doctors for further details about the health condition. Because of this technique the doctors can monitor their patient's health condition remotely. 


\section{Literature Survey}

$\mathrm{In}^{1}$ proposed the design of smart mirror based on raspberry pi. An intelligent is designed in such a way that raspberry pi is connected to the network through wifi. Then it will obtain information about the weather forecast, time, date and other information and then the information displayed on the plasma display. The user can interact with the mobile phone through the mirror. The advantages of intelligent mirror are small size, low cost, simple operation and also it is suitable for families also.

$\mathrm{In}^{2}$ also says about the design of smart mirror using Raspberry Pi. The study details about the design and development of an interactive multimedia futuristic smart mirror with AI for the home environment and also for commercial uses in different industries. Here also the interaction with the mirror is possible through speech processing technique.

$\operatorname{In}^{3}$ tells about smart mirror which can detect and monitor facial signs over time correlating them with cardio-metabolic risk and thereby providing guidance to users on how to improve their habits. The paper describes about the Europian project SEMEOTICONS to develop a device which detect and monitor facial signs and thereby giving personal guidance for the lifestyle. The clinical validation of wize mirror is ongoing. It focuses on the reproducibility of measurements provided by the wize Mirror and the correlation of estimated wellness with respect to cardio-metabolic risk charts.

$\mathrm{In}^{4}$ says about smart mirror which allows the user photos to access the mirror and then using authentication with facial recognition and user information to predict the news according to their profile. Authentications with facial recognition, customized news recommendation system and voice commands are the main added features to the existing system.

$\mathrm{In}^{5}$ proposed a multi user smart mirror system which promotes wellness and healthier life styles in the work environment. By using an RFID reader the mirror recognizes different user's through their personal ID card and there by allow to access a personalized user interface. The mirror will provide the environmental condition in the work place, the personal information obtained from wearable devices and the general information also. Employees can also access their ranking position from the mirror.

$\mathrm{In}^{6}$ present that IoT can play a significant role in remote health monitoring. In this study they proposed a system for monitoring the pulse rate and body temperature of a person by using dedicated sensors along with raspberry pi and IoT. Remote monitoring is achieved by storing the collected data to Bluemix cloud. Then the data can be accessed by the doctor from anywhere in the world. It uses temperature sensor and heart rate sensor to take the physiological data of the user.

$\mathrm{In}^{7}$ addressed the problem of proactive monitoring of one's health by recommending wearable technologies and IoT solutions that can be used by particular individual. This can be achieved by analyzing the individual's unstructured medical history using text mining, adding this data to structured demographic attributes and then giving this data to a machine learning classification model which predict the disease, and then mapping these diseases to the attributes. Lastly, it uses a mathematical optimization model to suggest the wearable devices to the user. $\operatorname{In}^{8}$ proposed an android based mobile data acquisition solution. It will collect the personalized health information of end user and then it store, analyze and visualize it on the smart devices.

The smart mobile device can collect information from various wirelesses and wired sensors. Embedded sensors in mobile device will provide useful status information also. It provides low complex, low power consumption and highly portable system for health monitoring.

$\mathrm{In}^{9}$ tells about the current trends and also the challenges and opportunities available in smart health care. They says that, by reducing the gap between researchers and health care professionals more research problems and diseases can be addressed and smarter life style can be adapted.

$\mathrm{In}^{10}$ tells about an architecture based on an ontology which is capable of monitoring the health and recommend eating habits and workout routines to patients. The people having diseases like diabetes, heart and pressure among others are more recommended in this technique.

$\mathrm{In}^{11}$ proposed a smart mirror which is used for home automation. It controls the mechanisms of home appliances and opening and closing of shelves through speech recognition techniques. It has some additional functionality, like reminder service by mobile synchronization and through social media.

$\mathrm{In}^{12}$ described about smart reflect which is a smart platform for developing smart mirror applications. The main features of smart reflect are, it is modular, lightweight and extensible. It allows developers to sidestep the 
sandboxed environment created by web browsers and it support plugins written in any programming languages.

In $^{13}$ compares smart sensors, smart objects and the things in internet of things. Both similarities and differences have been identified. Comparisons have been made for definitions provided by various authors and organizations.

In ${ }^{14}$ tells about a system designed to monitor the temperature and heartbeat of the patient using IoT. Then we can send the real time information to several users including doctors in critical situations and also we can use a buzzer to alert the care taker.

In $^{15}$ also described about the health monitoring system using IoT. The sensors will gather the medical information of the patient which include heart rate, blood pressure and pulse rate. Then it will be sent to the internet through Raspberry Pi. So doctor can monitor the health condition of the patient from any place of the world.

\section{Benchmarks}

The various related products are studied; their features, advantages and disadvantages are given in Table 1 .

\section{Methodology}

The concept of the smart mirror was got from people's daily device which includes PCs, smart phones, tablets etc. The mirror integrated the features from each of these devices. Figure 1 is a schematic view of the smart mirror concept.

Smart mirror is a simple concept with the aim of the mirror which provides an easy way for one to access information services such as news feeds weather etc. The main added feature is health monitoring and abnormality detection.

The Raspbian operating system is downloaded and the image is extracted on SD card. The card is inserted in the raspberry pi SD slot and then performing the required step. The biomedical sensors are added to the mirror surface. Here, sensors like temperature sensor, blood pressure sensor and heart beat sensor are used. These sensors will collect the physiological data of the user. The collected data was stored in the cloud. Here we used the Think Speak cloud. ThingSpeak is an IoT platform that will collect and store sensor data in the cloud and then it will help to develop IoT applications. The ThingSpeak IoT platform provides apps that help to analyze and visualize our data in MATLAB, and then act on the data. Most people have mirrors at home, so the concept of a smart mirror that you can interact with is attractive.

Nowadays the sensors are there within products like mobile phones, watches etc. which are seldom used by old aged people. So this paper introduces health monitoring through mirrors which can be made use by people irrespective of their ages. The information collected by the biomedical sensors present in the smart mirror will be sent to the doctors so that they can monitor the health conditions of their patients remotely.

Table 1. Features, advantages and disadvantages of health products

\begin{tabular}{|l|l|l|l|}
\hline Product & Features & Advantage & Disadvantage \\
\hline Magic Mirror & $\begin{array}{l}\text { It comes with the basic } \\
\text { widgets for time, calendar, } \\
\text { weather and news }\end{array}$ & $\begin{array}{l}\text { It looks very good and has a clean user } \\
\text { interface }\end{array}$ & $\begin{array}{l}\text { It has no physical interaction } \\
\text { with the user and simply act as } \\
\text { an information panel }\end{array}$ \\
\hline Home Mirror & $\begin{array}{l}\text { It also shows useful } \\
\text { information like time, } \\
\text { weather, date and reminders }\end{array}$ & $\begin{array}{l}\text { It uses an android tablet behind the } \\
\text { mirror. So no need of a separate } \\
\text { computer board and screen }\end{array}$ & $\begin{array}{l}\text { It also lacks any kind of } \\
\text { interaction. }\end{array}$ \\
\hline $\begin{array}{l}\text { Evan Cohen's smart } \\
\text { mirror }\end{array}$ & $\begin{array}{l}\text { It offers a wide range of voice } \\
\text { commands to interact with } \\
\text { the mirror }\end{array}$ & $\begin{array}{l}\text { The voice commands support time, } \\
\text { date and weather information, } \\
\text { showing maps, adding reminders etc. }\end{array}$ & $\begin{array}{l}\text { Voice commands cannot be } \\
\text { sufficient to control other } \\
\text { devices like home automation. }\end{array}$ \\
\hline $\begin{array}{l}\text { Max Braun's Smart } \\
\text { Mirror }\end{array}$ & $\begin{array}{l}\text { It will update the useful } \\
\text { information automatically. }\end{array}$ & $\begin{array}{l}\text { It support voice commands to perform } \\
\text { Google search }\end{array}$ & $\begin{array}{l}\text { It has a small level of } \\
\text { interactivity. But still basically } \\
\text { an information panel only. }\end{array}$ \\
\hline PANL & $\begin{array}{l}\text { It is one of the first touch } \\
\text { screen smart mirror }\end{array}$ & $\begin{array}{l}\text { It can display useful information like } \\
\text { time, date, weather and also can play } \\
\text { music, you tube videos etc. }\end{array}$ & $\begin{array}{l}\text { The founder not revealed much } \\
\text { information about the hardware } \\
\text { and software part of the mirror. }\end{array}$ \\
\hline
\end{tabular}




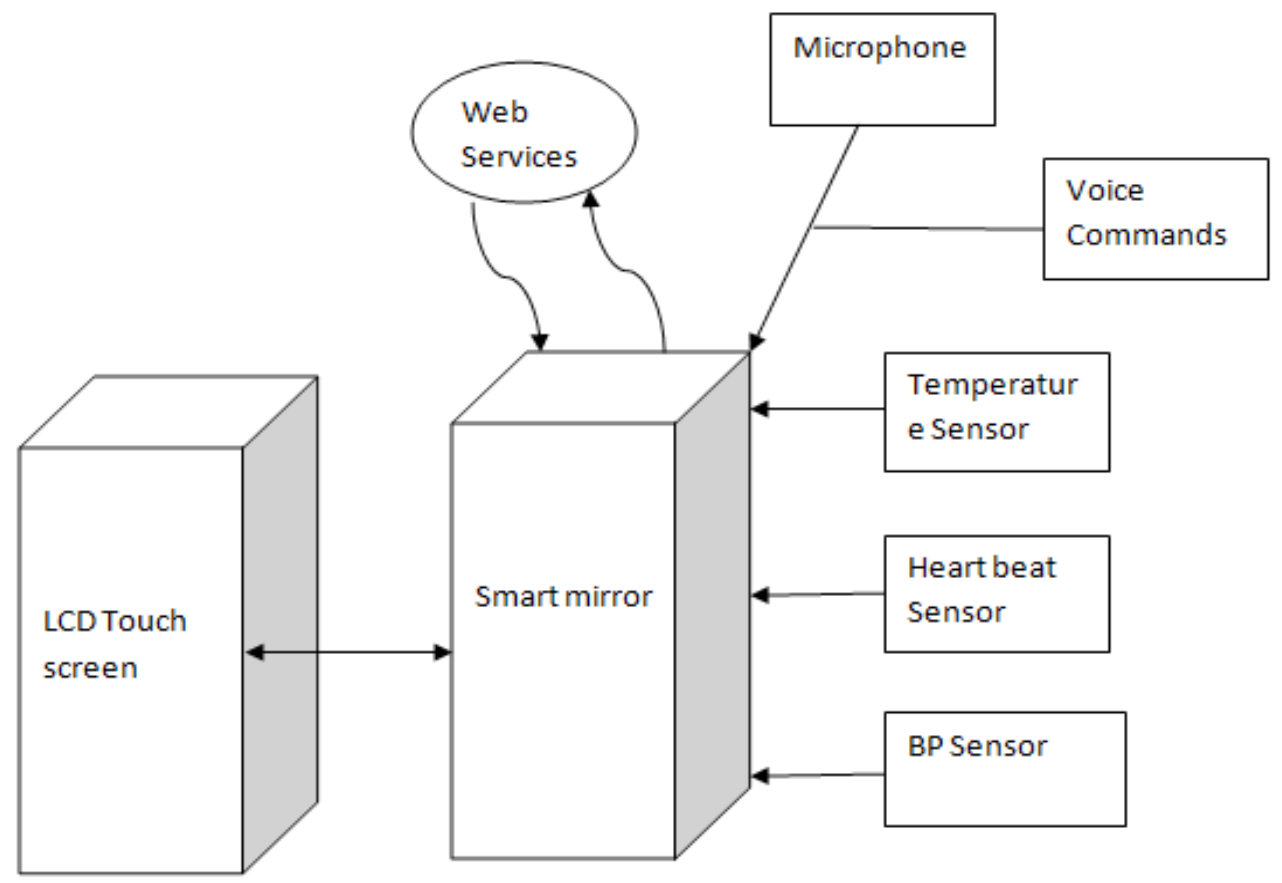

Figure 1. Schematic view.

The Smart Mirror CPU is the Raspberry Pi 3 computer on which all the software components would lie in. The processor takes in video information from the camera, After the CPU identified the person standing in front of it, it would retrieve the information programmed to show for that user. Then the CPU projects this information on to the connected LCD Screen. The users can touch the biomedical sensors for getting their physiological information. The data is displaying on the connected LCD screen and also it stores the data in the cloud for further monitoring of user's health by the experts. The block diagram of the smart mirror for health monitoring and abnormality detection is shown in Figure 2.

\section{Components}

\subsection{One Way Mirror}

A one-way mirror, sometimes called two-way mirror is a mirror which is partially reflective and partially transitive. The dark or black side of the screen is seen as a reflection and the light parts seen normally.

\subsection{Display}

The smart mirror needs a display which will be connected to the two-way mirror. Now TV screens are using as display .But in a cost effective way we can use tablet as the screen. An HDMI cable is used to connect the display with Raspberry Pi.

\subsection{Raspberry Pi}

The Raspberry Pi is a single-board computer developed by Raspberry foundation in UK. It lacks a hard drive and it does not have any preinstalled OS.To install the OS it need a micro SD card.

\subsection{Microphones}

To interact with the mirror it uses microphones. Two microphones are using to power the voice recognition capabilities. The first microphone is a simple one connected through a USB sound card to the Pi. The second microphone is a PS3 eye camera which can directly connect through USB. 


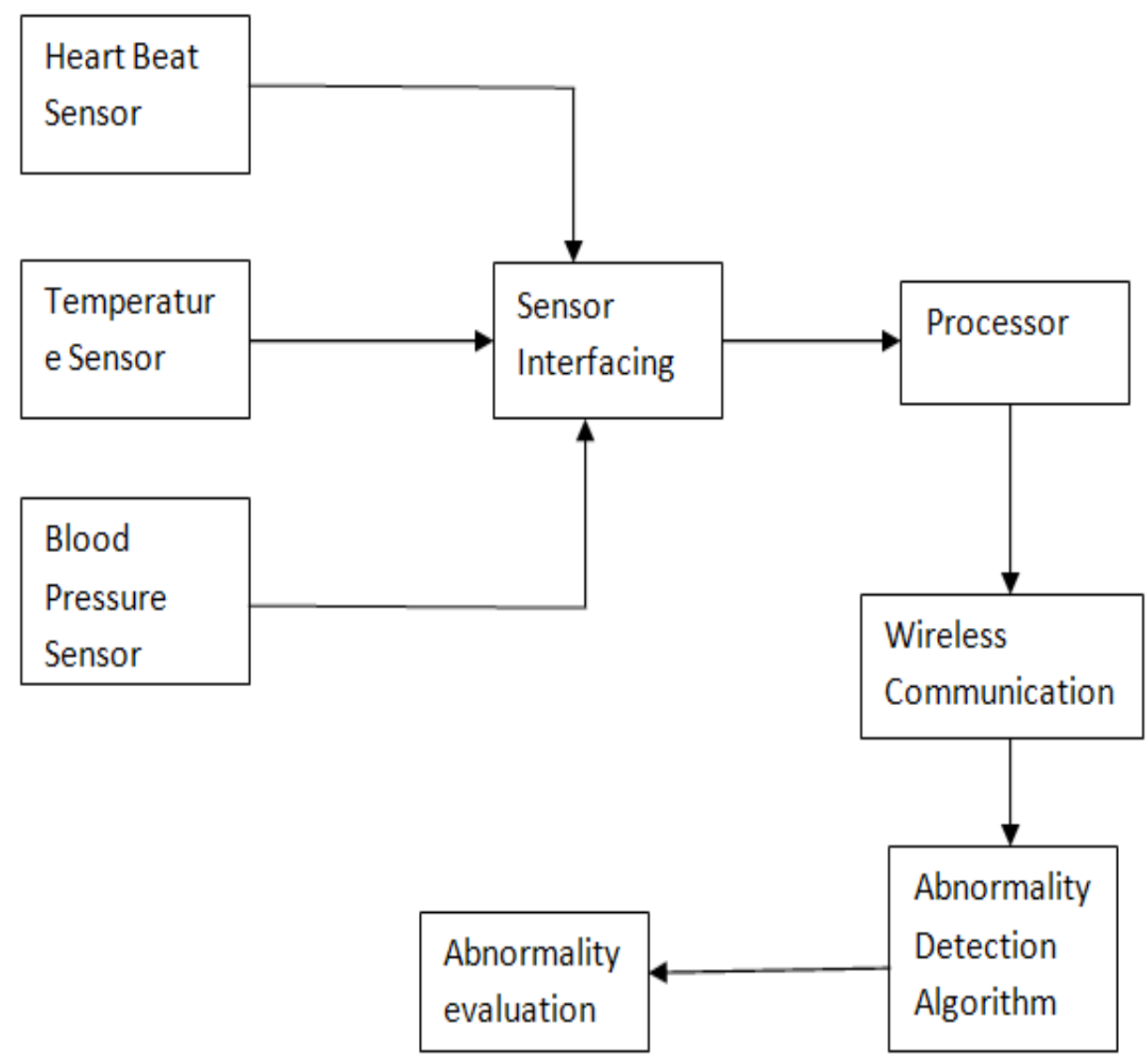

Figure 2. Smart mirror block diagram.

\subsection{Frame and Support}

The Frame is made of wood and it provides support for mirror and all other components.

\subsection{Sensors}

The system uses different biomedical sensors to read the human physiological information in order to monitor the health condition.

The hand- held ECG sensor is used to provide the ECG biofeedback information. The result is used to monitor the heart activity patterns of the user. Measuring the stress level is one of the objectives of recording the ECG signal.

The blood pressure sensor measures the systemic arterial pressure measured at the user's finger. It will find the blood flow and thereby able to detect the blood volume changes in the finger.

A skin temperature sensor will measure the palm temperature of the user. There are such different sensors are available that are capable to measure the skin temperature and humidity. Such meters will increase the understanding of user's health condition.

Weight meter is also used to measure the weight of user continuously. The readings obtained are helpful to those who are trying to lose weight.

\section{Implementation}

The development of Health Monitoring System has been motivated mainly by the increasing healthcare costs and by the fact that the world population is ageing. So, to make easy monitoring and abnormality detection, a new platform was developed for health sectors which monitors different parameters on health at home and detects various diseases.

For that we use IoT based devices which collect the medical data of the user. The UCI Repository also has data for heath monitoring. Medical dataset consists of history of the patient data. All these datasets are stored 
into cloud database. These data will be stored securely in cloud based environment. by performing five different stages of a new storage mechanism such as data storage in to cloud, data retrieval from cloud database, data aggregation, data partitioning and finally data merging. Then the health analysis and finding the abnormality is done using another algorithm. Finally, the patient records are classified as normal and abnormal.

The proposed cloud based health monitoring and abnormality detection consists of three phases. First phase is used to collect the necessary data from different biomedical sensors, UCI Repository and history of patient health details. Second phase is used to store these records on cloud database. Third phase is responsible for detecting the abnormalities.

\subsection{Data Collection}

This phase is responsible for collecting the individual patient detail which extracted by using the sensors like temperature sensor, heart beat sensor, blood pressure sensor attached to the mirror surface. The UCI repository dataset values are used for mapping with the actual data and also the hospital medical records are used for mapping with the actual these data.

\subsection{Data Storage on Cloud Database}

The system considered three different kind of medical data for decision making. First one is the data collected from the sensors. Second is the UCI repository dataset. Third is the medical history of the user received from the hospital. These three different datasets must be stored in a database for analysis. The cloud environment provides sufficient space for storing this huge amount of data. It will be stored in Hadoop which will provide scalability. But the data security is a challenge in a cloud environment. For this purpose, we use a new storage algorithm. The storage algorithm works in two phases namely storage phase and retrieval phase.

\subsubsection{Phase 1: Storage Phase}

Input: Input Data

\section{Output: Numeric Data}

1. Get the medical details as input.

2. Find the ASCII values for the input data in numeric form

3. The numeric values should be expressed as a matrix.
4. For each matrix element do

- Each element will be replaced with numbers which are sum of all the elements which are present in the respective row and column.

- Call aggregation procedure for placing the elements in the correct place.

- Call AES algorithm for encrypting the matrix data.

\subsubsection{Phase 2: Retrieval Phase}

Input: Numeric Data

Output: Decrypted Medical Data

1. Perform decryption operation for the matrix values by applying the respective keys and the algorithms.

2. Perform the subtraction operation on all the elements present in the respective rows and columns in order to get the original value from the summation values.

3. Print the resulted values as output

\subsubsection{Phase 3: Data Aggregation}

Input: Summation values for the medical data Output: Aggregated data

1. Read the summation values.

2. All these input values will be converted into the respective numbers of the data using look up table and it wll be stored in another table NTab.

3. For each element of NTab.

- Summation values for all the particular row elements of NTab will be calculated.

- Summation values for all the particular column elements of NTab will be calculated.

4. Aggregated values will be stored into the correct position of the table NTab in a table Aggregation that is secured.

\subsection{Abnormality Detection}

The algorithm depends on the step by step procedure of the calculations, detection of different the parametric value and then detects the abnormality. Starting at initial input, it will perform the computation, execution, process, and it will successively produce output and terminating at final state. The data is read from bio medical sensors. Based on the data, the abnormality is detected in the health condition. If there is any deviation in the health condition, the corresponding details are sent to the doctor for further medication. 


\section{Conclusion}

The designed smart mirror facilitates the health monitoring for the people using different biomedical sensors which collect different physiological features like heartbeat, blood pressure, body temperature. The collected data was analyzed to find the abnormalities. If there is any abnormality in the data, it will be sent to the corresponding doctors for further detailing.

\section{References}

1. Sun Y, Geng L, Dan K. Design of Smart Mirror Based on Raspberry Pi. International Conference on Intelligent Transportation, Big Data \& Smart City (ICITBS), 2018. p. 77-80. https://doi.org/10.1109/ICITBS.2018.00028.

2. Hossain MA, Atrey PK, El Saddik A. Smart mirror for ambient home environment. IET International Conference on Intelligent Environments; 2007. p. 589-96. https://doi. org/10.1049/cp:20070431.

3. Henriquez P. Mirror mirror on the wall... an unobtrusive intelligent multisensory mirror for well-being status self-assessment and visualization, IEEE Transactions on Multimedia. 2017; 19(7):1467-81. https://doi.org/10.1109/ TMM.2017.2666545.

4. García ICA. Implementation and Customization of a Smart Mirror through a Facial Recognition Authentication and a Personalized News Recommendation Algorithm. International Conference on Signal-Image Technology \& Internet-Based Systems; 2017. p. 35-39. https://doi. org/10.1109/SITIS.2017.17.

5. Gomez-Carmona O, Casado-Mansilla D. SmiWork: An interactive smart mirror platform for workplace health promotion. International Multidisciplinary Conference on Computer and Energy Science (SpliTech); 2017. p. 1-6.

6. Health monitoring based on IoT using Raspberry PI. Date accessed: 6.05.2017. https://ieeexplore.ieee.org/document/8230004.

7. Asthana S, Megahed A, Strong R. A recommendation system for proactive health monitoring using IoT and wearable technologies. IEEE International Conference on AI \& Mobile Services (AIMS); 2017. p. 14-21. https://doi. org/10.1109/AIMS.2017.11.

8. Lakshmanachari S. Design and implementation of cloud based patient health care monitoring systems using IoT. International Conference on Energy, Communication, Data Analytics and Soft Computing (ICECDS); 2017. p. 3713-17. https://doi.org/10.1109/ICECDS.2017.8390157.

9. Sundaravadivel P. Everything you wanted to know about smart health care: Evaluating the different technologies and components of the internet of things for better health, IEEE Consumer Electronics Magazine. 2018; 7(1):18-28. https:// doi.org/10.1109/MCE.2017.2755378.

10. Gomez J, Oviedo B, Zhuma E. Patient monitoring system based on internet of things, Procedia Computer Science. 2016; 83:90-97. https://doi.org/10.1016/j. procs.2016.04.103.

11. Athira S. Smart mirror: A novel framework for interactive display. International Conference on Circuit, Power and Computing Technologies (ICCPCT); 2016. p. 1-6. https:// doi.org/10.1109/ICCPCT.2016.7530197.

12. Gold D, Sollinger D. SmartReflect: A modular smart mirror application platform. IEEE 7th Annual Information Technology, Electronics and Mobile Communication Conference (IEMCON); 2016. p. 1-7. https://doi. org/10.1109/IEMCON.2016.7746277.

13. Liu X, Baiocchi O. A comparison of the definitions for smart sensors, smart objects and Things in IoT. IEEE 7th Annual Information Technology, Electronics and Mobile Communication Conference (IEMCON); 2016. p. 1-4.

14. Sivakanthand TS, Kolangiammal S. Design of IoT based smart health monitoring and alert system, International Journal of Control Theory and Applications. 2016; 9(15):7655-61.

15. Kumar R, Rajasekaran MP. An IoT based patient monitoring system using raspberry $\mathrm{Pi}$. International Conference on Computing Technologies and Intelligent Data Engineering (ICCTIDE'16); 2016. p. 1-4. https://doi.org/10.1109/ ICCTIDE.2016.7725378. 